\title{
A Multimodal Analysis of Hospital Homepages
}

\author{
Lingwei Kong, Southern Medical University
}

\begin{abstract}
The growingly wide use of hospital homepages extends patient resources locally and internationally. This study, adopting the socio-semiotic multimodal approach (Kress, 2010; Bezemer \& Kress, 2016), examines the multimodal features of two hospital homepages which were collected from University College London Hospitals (UCLH) in London, the UK and Nanfang Hospital $(\mathrm{NH})$ in Guangzhou, China. The study reveals how different modes are exploited in the construction of hospital homepages and how mode applications are influenced by professional practices and socio-cultural constraints.
\end{abstract}

Keywords: mode; multimodality; social semiotics

\section{Introduction}

The expansion of Internet environment has produced new genres. Homepages, presenting typical examples of such new genres, work as 'the top-level document of a web site' (Askehave \& Nielsen, 2005:123), which functions as official gateway to establish public images for companies and institutions. The use of the Internet as a channel for marketing and communication in medical services is mushrooming. The growingly wide use of hospital homepages presents both an exciting challenge and a big niche pertaining to how to communicate with existing patients and how to draw attention from new patients locally and internationally.

Mode is 'a socially shaped and culturally given semiotic resource for making meaning' (Kress, 2010:79). The meanings of a multimodal text could not be generated 'if only one mode were to be used' (Bowcher, 2007:242). Multimodality pertains to conditions in which at least two input (senses) or output (medium/device) modes are involved (Pauwels, 2012:250).

Some studies have been carried out on multimodal texts online for medical services (Sanchez \& Fuentes, 2002; Usher \& Skinner, 2010). There are a variety of studies on mode applications to websites or homepages, describing different modes, such as writing (Kong, 2014; Lam, 2013), image (Garrick \& Pendergast, 2014; Michelson \& Valencia, 2016), and sound (Pennock-speck \& Saz-Rubio, 2013). However, it is very rare to find any research zooming in modes of online multimodal texts via making comparison analyses inter-culturally. Aiming to bridge the gap, the present study, focusing on key modes, examines two hospital homepages collected from the UK and China respectively.

The present study aims to address the following research questions: 1 . How are different modes exploited in the construction of hospital homepages? 2. How are mode applications in constructing hospital homepages influenced by professional practices and socio-cultural constraints?

\section{Social semiotics and multimodality}

Social semiotics is considered as 'a synthesis of several approaches to the study of social meaning and social action' (Lemke, 1990:183), expanding the research scope of formal semiotics to disclosing how social resources are exploited to encode the meaning and how signs are used to construct the life of a community. Text is 'multimodal' (Hallett \& Kaplan-Weinger, 2010:118) within 'specific social contexts, and in ways which are appropriate to the interests of social actors in these contexts' (van Leeuwen, 2005:94).

The social semiotic multimodal approach provides theoretical tools to examine multimodality, which is like 'a fork with two prongs', namely 'the semiotic and the multimodal prong' (Kress, 2010:105). The first prong attends to the social semiotic, dealing with 'how signs are made; how meaning is shaped; what discourses and what genres are available and how they 
are used; what texts are and how they work; how representation and communication function; that modes occur in ensembles' (ibid.:105). The second prong attends to the multimodal, focusing on 'what the potentials of each mode in these ensembles are, the fact that modesimage, architecture, speech, gesture- have specific affordances and differing semiotic meansdiffering means for framing, for instance' (ibid.:105). In essence, the socio-semiotic multimodal approach combines the way examining semiotic work and social environments.

\section{The present study}

The present study, drawing on the socio-semiotic multimodal approach (Kress, 2010; Bezemer \& Kress, 2016), examines modes in ensembles in hospital homepages.

\subsection{Data}

Medical services represent a typical setting involving negotiation of different cultural values and practices. As renowned international metropolises, both London and Guangzhou take significant roles in international business interactions, and thus form ideal research sites for examining intercultural and global professional communication.

The study focuses on two homepages respectively from University College London Hospitals (UCLH) in London, the UK and Nanfang Hospital (NH) in Guangzhou, China. The screenshots of the homepages were saved considering the dynamic changes that can take place online. Live pages were used when it was necessary to examine some functions and determine hyperlink references in the homepages. Appendices 1-5 and Appendices 6-8 are two sets of examples of screen shots of UCLH and NH homepages, all of which were collected on 22 February 2016.

\subsection{Integrated approach}

Modes tend to appear in ensembles (Bezemer \& Kress, 2016:7). The study thus adopts an integrated approach to examine modes via combining a set of theories from the social semiotic view which include functionality of layout (Kress, 2010), visual grammar (Kress \& van Leeuwen, 1996, 2006), and image-text links (van Leeuwen, 2005). The study also examines text-external resources - professional practices and socio-cultural constraints which shape the multimodal texts.

Firstly, the study examines layout, which refers to 'the arrangements of elements on a site of appearance' (Kress, 2010:90). Kress (ibid.:88) proposes three questions to examine how layout functions as a mode:

1. Can layout form message-entities which are internally coherent and which cohere with their environment?; 2. Can layout represent meanings about the social relations of those engaged in communication?; and 3. Can layout represent meanings about the world of states, actions and events?

The three questions substantiate the conceptions of the three meta-functions, following Halliday (1994), namely textual, interpersonal and ideational. Attempting to test the questions, the study zooms in layout by examining the three meta-functions respectively.

Secondly, visual grammar proposed by Kress and van Leeuwen $(1996,2006)$ is adopted to analyse the images of the homepages. Represented participants and shots and social distance are analysed in detail. Spatial distance reflects social distance, suggesting the relation between the human represented participant in the image and viewers (Kress \& van Leeuwen, 1996, 2006; Machin, 2007). A close shot is used to represent intimate social distance, through which the personality of the represented participant could be more clearly observed; however, the represented participant in a far distance tends to lose individuality and become a stranger to the readers (Kress \& van Leeuwen, 1996, 2006).

Thirdly, the study discusses about writing by examining image-text relations, adopting image-text linking system and focusing on the occurrences of two major relations, namely 'elaboration' and 'extension' (van Leeuwen, 2005:230). In the case of elaboration, the 
information offered by Mode A restates the information offered by Mode B for purposes of clarification. In the case of extension, Mode A adds new information, linking it to the existing information offered by Mode B. Elaboration could be achieved by specification or explanation. Extension is realised by similarity, contrast, and complement.

\section{Social semiotic multimodal analysis}

The UCLH and NH homepages present a set of similar and differential characteristics in mode applications. This section discusses key modes occurring in the two homepages.

\subsection{Layout}

\section{a. On textual function}

The UCLH and NH homepages consist of a variety of modes, among which the major ones are layout, image and writing. All these semiotic resources are spatially arranged into salient units termed modules at a macro-level producing the homepages as semiotic entity.

Module, as a 'catch-all term', categorises the units which compose texts or semiotic entities (Kress, 2010:147). The ten modules of the UCLH homepage, as shown in Appendices 1-5, termed as Modules 1-10, present separate sections which parallel one another and are vertically positioned in space. Module 1 communicates the basic information about medical services and professionals at UCLH; Module 2 presents stories of people involved in medical services including hospital staff and patients; Module 3 provides guidance tools to readers; Module 4 elaborates 'What's new at UCLH'; Module 5 presents 'Patient safety' at UCLH; Module 6 provides the information of 'Work with us' at UCLH; Module 7 elaborates 'How are we doing' and describes professional services offered by UCLH; Module 8 guides potential patients to appropriate sections of medical services; Module 9 outlines 'Future developments' of UCLH; and Module 10 presents institutional values of UCLH and provides 'Contact us' methods. The ordering arrangement of the ten modules is not random. They are positioned to develop a coherent storyline, sequentially introducing 'who are we' (Modules 1-3), 'what are we doing' (Modules 4-8), and 'what will we be doing in the future' (Module 9), and in the end highlighting 'how to contact us'. Any re-arrangement of the modules might ruin the internal coherence of the text and change the meaning.

$\mathrm{NH}$ homepage has six modules. Module 1 provides a set of hyperlinks leading to detailed information about $\mathrm{NH}$ and generalising the information which occur in the other modules; Module 2 presents basic information about all the departments of the hospital; Module 3 shows a set of hyperlinks as shortcuts which lead to relevant medical services of the hospital; Module 4 elaborates 'What's new at NH'; Module 5 uses a set of hyperlinks which link to other medical institutions and enrich medical services sources for readers; and Module 6 presents institutional core values and provides 'Contact us' methods and recruitment information.

The six modules tend to arrange messages in the order of significance. Most readers approach the hospital homepage for the information about what professional medical services the hospital can provide to satisfy their individual needs. Modules One to Four, as shown in Appendices 6-8, positioned in the eye-catching places, on the top or in the central place of the homepage, present the core information about $\mathrm{NH}$ medical services and professional staff. It saves readers' efforts to search information. If readers cannot find what they want, Module 5 also gives them help by listing a set of hyperlinks connected to other medical institutions. The six modules do not indicate any storyline; nevertheless, they co-work to achieve quick and efficient guidance which favours readers to accomplish their information search. The motivation, considering patients' needs as core value of medical services, goes through all the modules, which evidently responds to the NH's service principle, namely 'patients oriented, life respected', as shown in Module 5. If the arrangement of the modules alters, the internal coherence of the homepage will be changed. The modules, well organized to achieve the textual coherence, thus help to achieve the textual function of the multimodal text. 


\section{b. On interpersonal function}

In the UCLH homepage, all the four major images in Module 2 and one major image in Module 6 are photographs, presenting the images of four hospital staff and one patient. The four photographs in Module 2 are positioned right to the direct quotations from the three hospital staff and the customer, and the major image in Module 6 is also in the right side of the writing 'Work with us', which all prioritise the images. Writing and images thus represent a typical leftto-right order (Kress, 2010), in which information in writing is assumed as already known knowledge to readers; while information in images is assumed as new knowledge to readers. The homepage producers incline to use the photos to send core messages and attract readers' attention.

In the NH homepage, the four major images presented as photos are positioned in the central place of the multimodal text. The images are centralized to highlight the core knowledge which homepage producers expect readers to get; while writing in surround attempts to provide the supportive information. This develops a centrality-marginality pattern for the relation of the image and writing (Kress, 2010).

It is interesting to find that producers of the two homepages, designing different places for images and writing to take, technically invite readers' attention and thus create interpersonal environment with readers. It highly suggests the interpersonal functions of layout.

\section{c. On ideational function}

The UCLH homepage mainly arranges writing and image in a left-to-right order, which indicates known information and new information respectively and can be regarded as a 'Western' style, denoting 'the taken-for-granted reading direction' (Kress, 2010: 92). Each module of the homepage is positioned in a vertical order which follows the traditional reading order in consuming reading materials.

The NF homepage locates large-sized images in the central place of the multimodal text. Writing is positioned in surround. Centrality of images and marginality of writing occur in the NF homepage to indicate the core information and marginal information. The six modules are not vertically positioned. They are arranged according to their significance in communicating core information. The modules delivering medical service information of $\mathrm{NH}$ are presented in the central place of the homepage. It also reflects how centrality is adopted to highlight core information.

The hypothesis that layout contributes to achieving the ideational function of multimodal texts is evidenced by the findings about the taken-for-granted reading direction in the UCLH homepage and the key-information-centered approach in the NH homepage.

\subsection{Image}

Images have the potential to capture and present the essence of things (Dillon, 2006). The major images on the UCLH and NH homepages were examined based on the visual grammar (Kress \& van Leeuwen, 1996, 2006).

Photography could be 'prized as an accurate and reliable transportation of real-world people and places' (Barrett, 1980:52), representing a high degree of facticity. All the five major images in the UCLH homepage and the four major images in the NH homepage are presented in the form of photos. The two homepages tend to use people or objects in real life to speak for their medical services,

The major images in the UCLH homepage present real individuals involved in medical services as represented participants, intended to shorten the distance between the medical institution and readers. The images presenting the real staff and real patient encourage readers to feel that they could also get friendly services from UCLH staff and enjoy the same satisfaction of the patient reflected in the images.

The major images in the NH homepage respectively present distinguished doctors of the hospital, the hospital main building, hospital management meeting and hospital core value system. Chinese patients are more inclined to choose medical institution based on what high- 
quality services the hospital can offer and what outstanding medical staff the service programme involves. The represented participants reflect what are highly expected by Chinese patients. An authority-respected theme is thus created in the images.

The photos in the UCLH homepage adopt the close shot to present represented participants. The facial expression of the medical staff and the patient can be clearly viewed, which creates a close personal distance between the represented participants of the images and readers.

The photos in the NH homepage use the medium close shot to present an outstanding professor as the represented participant, as shown in Appendix Six, which indicates a far personal distance between the represented participant and readers. Other two photos use long shot to present groups of hospital staff, which creates a close social distance between the represented participants and readers. In these photos, the homepage producers avoid emphasizing any individual in the images, thus 'impersonal business occurs' (Kress \& van Leeuwen, 2006: 124). The longer shots are the devices to reduce the 'degree' of 'individualization' (ibid.: 118), which also evidences the idea that collectivism is given priority in Chinese culture.

The UCLH homepage tends to create a close distance with patients via adopting close shots in images; however, the NH homepage attempts to foster an increased social distance which shapes the tone of images into a neutral and dispassionate one and encourages readers to interpret the meaning in an objective position.

\subsection{Writing}

Multimodal relations construct a complex interplay (Maier, 2008: 63). Two major types of image-text relations, following van Leeuwen's linking system (2005:230), were identified in the two homepages.

In the UCLH homepage, extension occurs and is achieved by complement via using the writing to add information to images. It highly suggests that images function as key modes in constructing the homepage. This image-text relation also resonates with the previous finding in Section 4.1 as indicated by the left-right order between writing and images in the UCLH homepage: the information in writing is assumed as already known knowledge to readers; while information in images are assumed as new knowledge to readers. The images function to enrich information of writing in the homepage.

In the NH homepage, elaboration occurs three times, which is realised by explanation. In this relation, the text paraphrases the image. Extension occurs once through the form of complement, in which the image adds information to the text. As discussed in Section 4.1, the images and writing are arranged in a centrality-marginality pattern in the NH homepage, which highlights the core information delivered by the images. Writing thus functions as a subsidiary support or paraphrasing of the images in this homepage.

The image-text relations suggest that images speak more than writing in the two hospital homepages, which might hint that hospital homepages have evolved into a type of multimodal texts highly depending on the presentation of images to attract more attentions from the readership. Images are adopted as key mode to retain readers for their online information searching.

\section{Conclusion}

The present study has recognized modes and their characteristics in two hospital homepages of the UK and China, which may further our understanding of production of online multimodal texts. Some interesting findings are as follows. Firstly, layout of hospital homepages is characterized by modules. Each module uses images and writing in ensembles. Modules have the potential to achieve three meta-functions, namely textual, interpersonal and ideational, contributing to forming multimodal texts in medical service contexts under the influence of core values of institutions and socio-cultural constraints. Secondly, photographs as major images are prevalent in the two homepages to represent a high degree of fact city. The 
represented participants of the images reflect the customer (patient)-oriented approach the hospitals take, aiming to satisfy different needs of patients in the two countries. Homepage producers show different tendencies to adopt a long or closer shot in creating images which suggests different types of social distance are involved in medical service contexts in the two countries. Thirdly, the pattern of image-text relation in the homepages highly suggests images tend to talk more than writing does in online multimodal texts and could be considered as a key mode to attract reading interest from the readership.

The present study has integrated a set of theories in the social semiotic view, namely functionality of layout, visual grammar and image-text links, to explore multimodal characteristics of homepages. This approach enables the understanding of the properties of modes in online multimodal texts, via considering professional practices and socio-cultural constraints, which hopefully might open more discussions on online multimodal texts in much wider range of occasions within different professional and cultural contexts. The present study has its limitations. It only examines two homepages from two countries. For future research, more homepages could be used as an ideal corpus which will produce more interesting and convincing findings and address more socio-culturally diverse traits.

\section{Funding}

This study was funded by Research Initiative Grant, Southern Medical University, 2014.

\section{References}

Askehave, I., \& Nielsen, A. E. 2005. Digital genres: a challenge to traditional genre theory. Information Technology \& People, 18(2), 120-141.

Barret, T. 1980. A structure for appreciating photographs. Exposure: The Journal of the Society for Photographic Education, 18(3/4), 50-55.

Bezemer. J., \& Kress, G. 2016. Multimodality, Learning and Commnication: A Social Semiotic Frame. New York: Routledge.

Bowcher, W. L. 2007. A multimodal analysis of good guys and bad guys in "Rugby League Week". In Royce T. D., \& Bowcher W. L (eds.). New Directions in the Analysis of Multimodal Discourse. Mahwah: Lawrence Erlbaum, 239-274.

Garrick, B., \& Pendergast, D. 2014. The impact of national agenda on a local education authority's website: a visual semiotic analysis. Cambridge Journal of Education, 44 (3), 299-317.

Hallett, R. W., \& Kaplan-Weinger, J. 2010. Official tourism websites: a discourse analysis perspective. Bristol: Channel View Publications.

Halliday, M.A.K. 1994. An Introduction to Functional Grammar (2nd edition). London: Edward Arnold.

Kong, L.W. 2014. A multimodal study of bank websites. Unpublished PhD thesis, The Hong Kong Polytechinic University.

Kress, G. 2010. Multimodality: a social semiotic approach to contemporary communication. London: Routledge.

Kress, G., \& van Leeuwen, T. 1996. Reading images: the grammar of visual design. London: Routledge.

Kress, G., \& van Leeuwen, T. 2006. Reading images: the grammar of visual design (2nd edition). London: Routledge.

Lam, P. 2013. Interdiscursivity, hypertextuality, multimodality: a corpus-based multimodal move analysis of Internet group buying deals. Journal of Pragmatics, 51, 13-39.

Lemke, J. 1990. Talking Science: Language, Learning, and Values. Norwood, NJ: Ablex Publishing Corporation.

Machin, D. 2007. Introduction to multimodal analysis. London: Hodder Education. 
Maier, C. 2008. Multimodal communication of specialized knowledge across hypertext innovation and generic tradition. Journal of Language and Communication Studies, 41, 59-80.

Michelson, K., \& Valencia, J. 2016. Study abroad: tourism or education? A multimodal social semiotic analysis of institutional discourses of a promotional website. Journal of Discourse \& Communication, 1-22.

Sanchez, A., \& Fuentes, M. 2002. Consumer orientation of public hospital websites in spain. International Journal of Medical Marketing, 3(1), 20-30.

Usher, W., \& Skinner, J. 2010. Categorizing health websites: e-knowledge, e-business and eprofessional. Health Education Journal, 70(3), 285-295.

Van Leeuwen, T. A. 2005. Introducing social semiotics. London and New York: Routledge.

Writing with images: Introduction: image text multiples and other mixed modes. Available at http://courses.washington.edu/hypertxt/cgi-

bin/12.228.185.206/html/wordsimages/wordsimages.htm. (Accessed on March 6, 2013). 


\section{Appendices 1-8}

Examples of UCLH and NH homepages
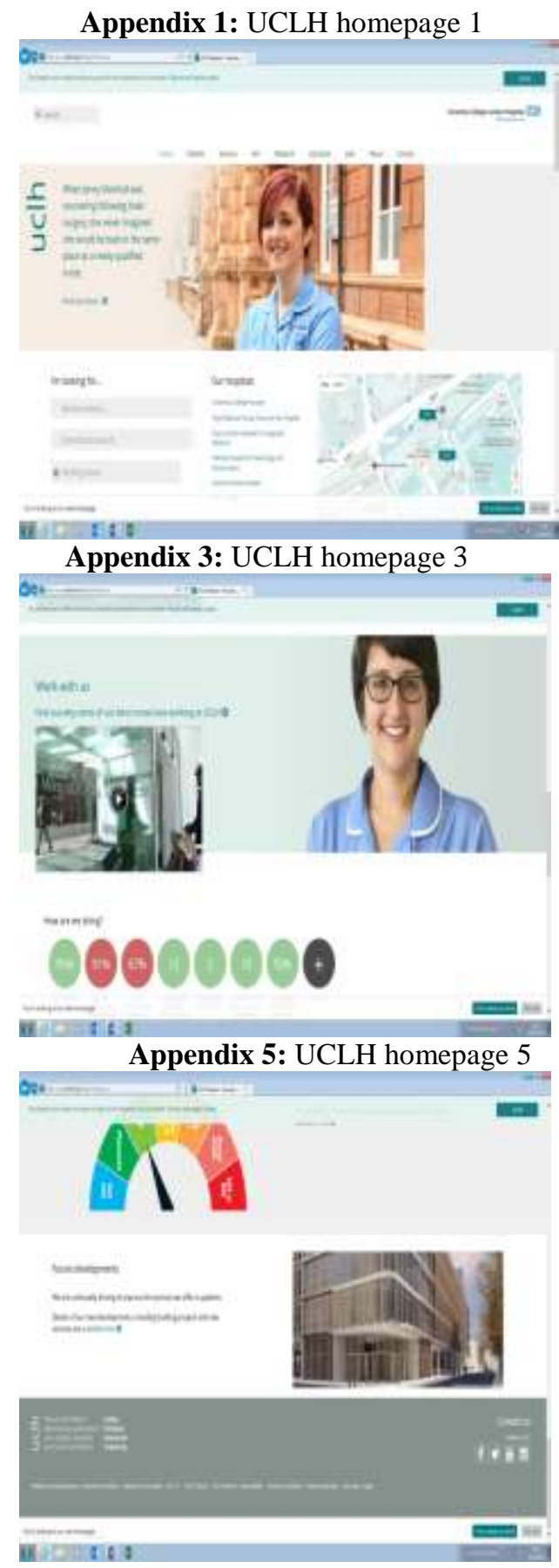

Appendix 2: UCLH homepage 2

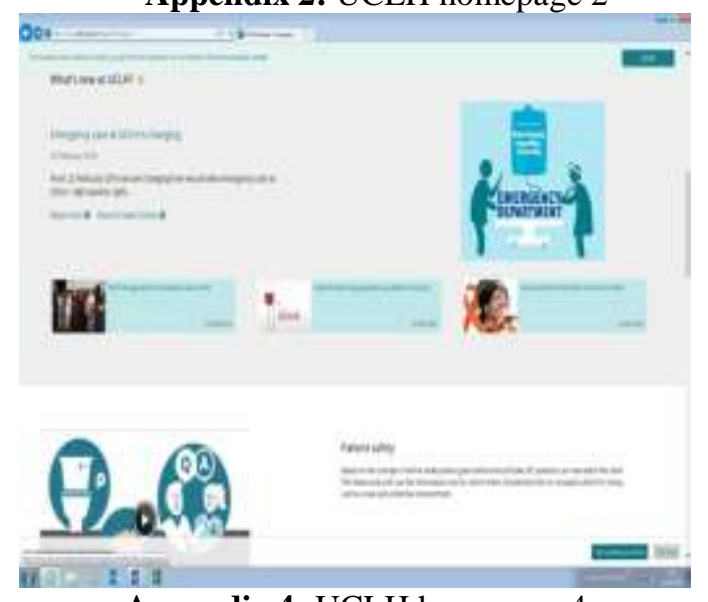

Appendix 4: UCLH homepage 4

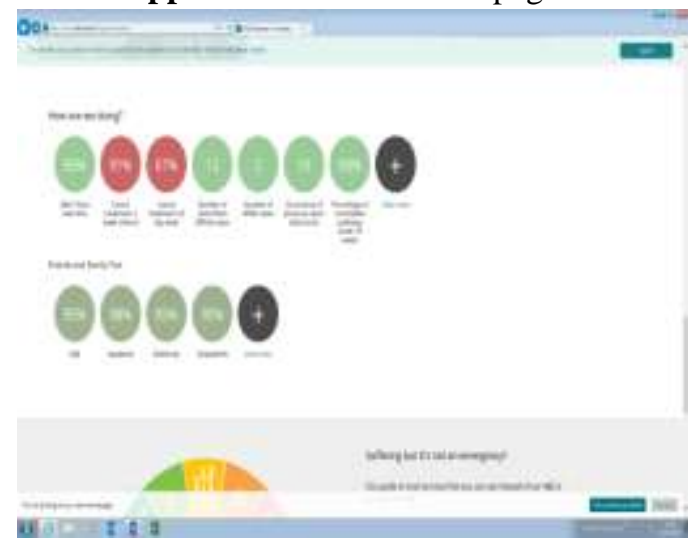

Appendix 6: $\mathrm{NH}$ homepage 1

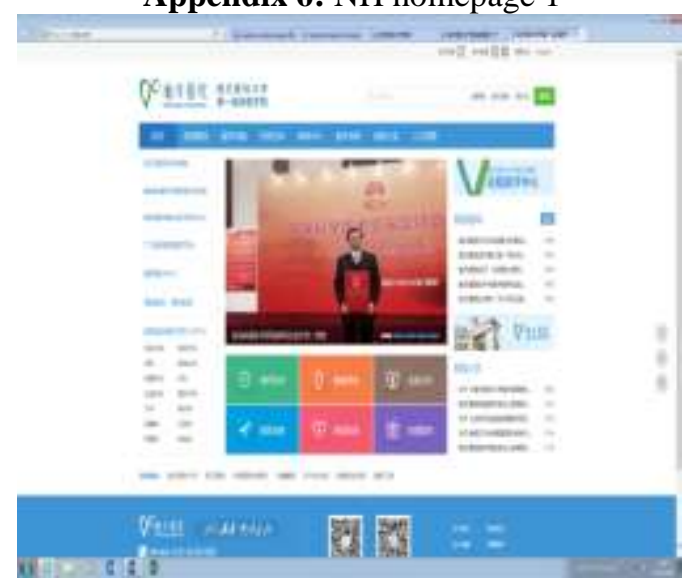


Appendix 7: $\mathrm{NH}$ homepage 2

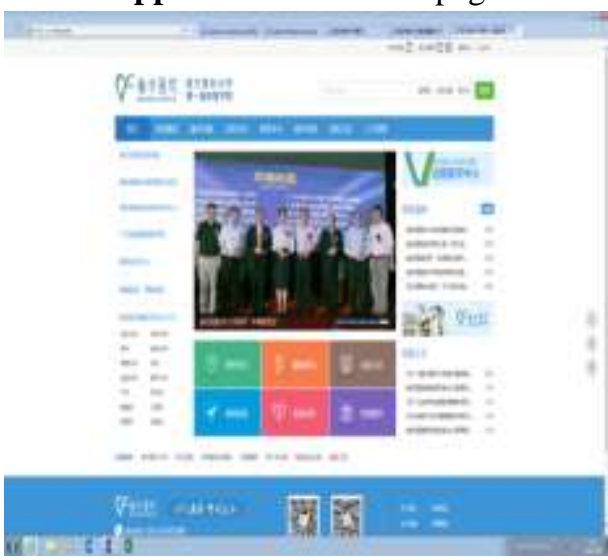

Appendix 8: $\mathrm{NH}$ homepage 3

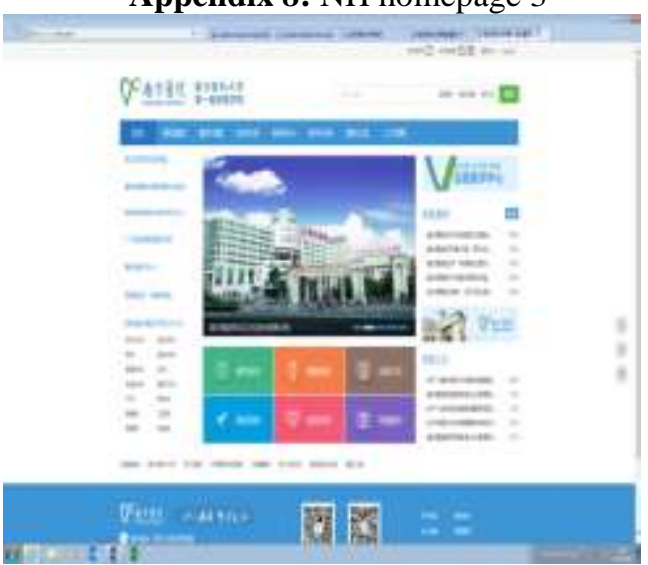

